\title{
Enhanced surface interaction of water confined in hierarchical po- rous polymers induced by hydrogen bonding.
}

\author{
Emilia V. Silletta ${ }^{1}$, Manuel I. Velasco', César G. Gómez², Siegfried Stapf ${ }^{3}$, Carlos Mattea ${ }^{3}$, Miriam \\ C. Strumia ${ }^{2}$, Gustavo A. Monti ${ }^{1}$ and Rodolfo H. Acosta*1. \\ 'FaMAF-Universidad Nacional de Córdoba and IFEG-CONICET, 5016 Córdoba, Argentina. \\ 2 Departamento de Química Orgánica, Facultad de Ciencias Químicas (IMBIV-CONICET), Universidad Nacional de Córdoba, \\ Haya de la Torre y Medina Allende, Edificio de Ciencias II - Ciudad Universitaria, 50oo Córdoba, Argentina.
}

${ }^{3}$ FachgebietTechnischePhysik II / Polymerphysik, Institute of Physics, Technische Universität Ilmenau, PO Box 100565,98684 Ilmenau, Germany

\section{SUPPLEMENTARY MATERIAL}

Longitudinal relaxation times are strongly dependent on the magnetic field for liquids confined in pore systems. Figure S1 shows three different T1 distributions for water contained in networks prepared with 33 mol \% of EGDMA content, which were acquired with different NMR equipment and deconvoluted using a numerical inverse Laplace transformation. Data corresponding at $2 \mathrm{MHz}$ were measured with a FFC spectrometer Stelar Spinmaster FFC20oo 1T/DC relaxometer, data at 6o $\mathrm{MHz}$ were acquired with a Keaz spectrometer from Magritek and data at $300 \mathrm{MHz}$ were acquired with a Bruker AvanceII spectrometer. All data are normalized to the same intensity before the ILT transform, changes in amplitude are due to the representation of the time axis in a logarithmic scale. As the area of each contribution $\left(\mathrm{P}_{1}, \mathrm{P}_{2}\right.$ and $\left.\mathrm{P}_{3}\right)$ remain constant for 2 and $60 \mathrm{MHz}$, the amplitudes change due to a shift of each relaxation time to higher values upon an increase in the magnetic field. At $300 \mathrm{MHz}$ the area corresponds to the addition of all contributions. At the time scale set at high frequency, NMR relaxation is given mainly by individual molecular rotations which do not provide information on the restricting geometry.

Figure S1. Longitudinal relaxation times dependence on the magnetic field. At high field a collapse into a single relaxation value close to bulk is observed. A polymer system prepared with $33 \%$ of EGDMA was measured.

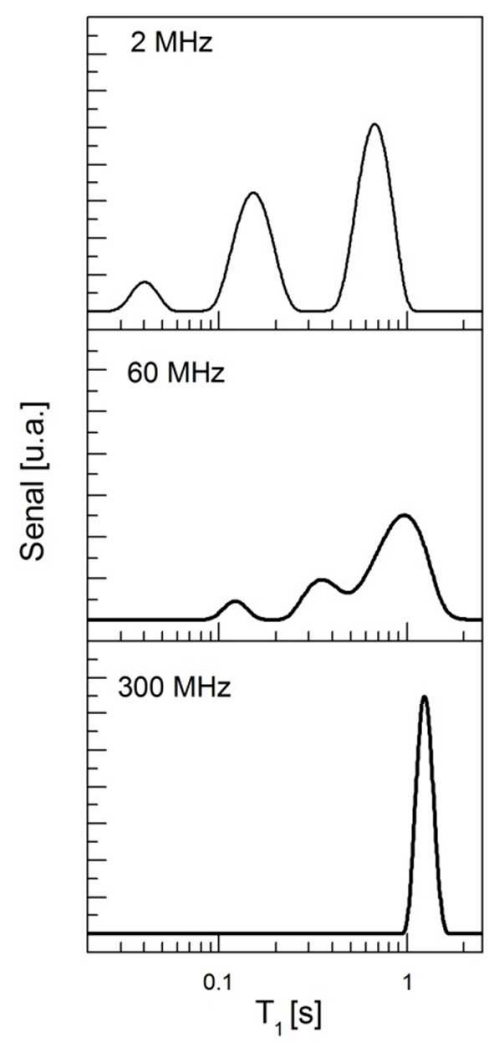

